\title{
Basilar Artery Territory Stroke Secondary to Invasive Fungal Sphenoid Sinusitis: A Case Report and Review of the Literature
}

\author{
Katherine A. Fu ${ }^{a, b} \quad$ Peggy L. Nguyen ${ }^{a} \quad$ Nerses Sanossian ${ }^{a, c}$ \\ ${ }^{a}$ Department of Neurology, ${ }^{b}$ Keck School of Medicine, and ${ }^{c}$ Roxanna Todd Hodges \\ Comprehensive Stroke Clinic, University of Southern California, Los Angeles, Calif., USA
}

\section{Key Words}

Stroke $\cdot$ Sinusitis $\cdot$ Mucormycosis

\begin{abstract}
Background: Mucormycosis is a fungal infection with the following 5 classic forms: cutaneous, pulmonary, gastrointestinal, disseminated, and rhinocerebral. The rhinocerebral form can be rapidly progressive and invasive with a high mortality rate. We present a case of a 38year-old man with invasive mucormycosis that led to a basilar artery territory stroke. Rhinocerebral mucormycosis is an unusual cause of stroke. Case Report: A 38-year-old man with a past medical history of diabetes mellitus presented with altered mental status. A lumbar puncture revealed eosinophilic pleocytosis with a mildly elevated total protein and borderline low glucose level. CT revealed a left medullary and cerebellar infarct confirmed by MRI. MRI also displayed a diffuse marrow signal abnormality in the clivus with contiguous sinus disease. Endoscopic sinus surgery confirmed that the fungal sinusitis was mucormycosis of the Rhizopus genus, which had affected the left sphenoid sinus, invaded through the skull base, and involved the basilar artery. He was given liposomal amphotericin (500 mg i.v.) with posaconazole (400 mg i.v. twice daily). Due to the severity of the invasion and poor prognosis, the patient was discharged with comfort care measures. Discussion: Clinicians should be aware of invasive sinusitis as a rare cause of stroke in diabetics. Once the subarachnoid space and basal arteries of the brain have been invaded, the prognosis is very poor. The key to improvement of outcomes is early recognition and treatment, and examination of the sinuses on neuroimaging in all cases of stroke is vital.


Fu et al.: Basilar Artery Territory Stroke Secondary to Invasive Fungal Sphenoid Sinusitis: A Case Report and Review of the Literature

\section{Introduction}

Mucormycosis is a saprophytic, filamentous fungal infection caused by Zygomycete fungi of the family Mucoraceae, and includes the genera Rhizopus, Rhizomucor, Mucor, and $A b$ sidia. Five classic forms of mucormycosis are recognized, including cutaneous, pulmonary, gastrointestinal, disseminated, and rhinocerebral [1]. Rhinocerebral mucormycosis is characterized by paranasal sinusitis, facial pain with swelling, headache, fever, rhinitis, granular or purulent nasal discharge, nasal ulceration, epistaxis, hemiplegia or stroke, and decreased mental function. Fungal invasion, often originating from the paranasal sinuses, leads to intracranial growth usually along the base of the skull. The rhinocerebral form is the most severe and may involve a rapidly progressive, invasive infection with a high mortality rate [1]. Rhinocerebral mucormycosis frequently occurs in immunosuppressed patients, and diabetes mellitus is often a predisposing risk factor for rhinocerebral mucormycosis [2]. We report the case of a 38-year-old man with a history of diabetes mellitus presenting with invading fungal sinusitis and fungal meningitis from mucormycosis that led to an ischemic stroke of the left hemi-midbrain, upper pons, and left cerebellum.

\section{Case Report}

A 38-year-old man with a past medical history of hypertension, chronic sinusitis, and diabetes mellitus presented with altered mental status. The patient reported fatigue during the past week; he denied having had fevers, chills, cough, shortness of breath, chest pain, and nausea or vomiting. On the initial evaluation, he was noted to have increased somnolence, with a Glasgow Coma Scale score of 11. Physical examination on admission was without significant findings. His vital signs were a temperature of $96.5^{\circ} \mathrm{F}$, a pulse of $76 / \mathrm{min}$, a respiratory rate of $16 / \mathrm{min}$, and blood pressure of $120 / 66 \mathrm{~mm} \mathrm{Hg}$. His pupils were equally round and reactive to light, his mucus membranes were moist, and a small laceration on the nose was noted. Both lungs were clear on auscultation, his heart sounds had a regular rhythm, and the heart rate showed normal S1 and S2 sounds and no murmurs, clicks, rubs or S3 or S4 heart sounds. He had 2+ pulses bilaterally and warm, dry skin. The abdomen was soft with an obese habitus, positive bowel sounds and no hepatosplenomegaly.

He was intubated for airway protection and given vancomycin $1 \mathrm{~g}$ i.v. every $12 \mathrm{~h}$, ceftriaxone 2 g i.v. every 12 h, and acyclovir 700 mg i.v. every 8 h. The noncontrast head CT performed on the day of admission revealed no intracranial mass, shift or bleed, but displayed a high density within the left frontal sinus. Lumbar puncture revealed eosinophilic pleocytosis (white blood cell count $400 \times 10^{9} / \mathrm{l}$ ), mildly elevated total protein $(63 \mathrm{mg} / \mathrm{dl}$ ), and borderline low glucose levels $(117 \mathrm{mg} / \mathrm{dl})$. Additional lumbar puncture results and cerebrospinal fluid (CSF) values are presented in table 1. Urine examination gave the following results: protein $300 \mathrm{mg} / \mathrm{dl}$, glucose $500 \mathrm{mg} / \mathrm{dl}$, and negative ketones, bilirubin, blood, nitrite, and leukocytes. Laboratory values revealed a high blood glucose level of $218 \mathrm{mg} / \mathrm{dl}$ and an $\mathrm{HbA}_{1 \mathrm{c}}$ level of $7.2 \%$. His wife reported that he had been taking glipizide and metformin for his diabetes, but could not recall any further information regarding his medications. According to the clinical examination and biochemical results, suspicion was highest for fungal and mycobacterial meningoencephalitis. West Nile virus (WNV) infection was also considered. Fluconazole 400 mg i.v. was initiated to cover for possible fungal infection.

Neurologists were consulted and the neurological examination was remarkable with a National Institutes of Health Stroke Scale (NIHSS) score of 12 . The patient appeared stuporous, opened his eyes to verbal stimuli, and followed commands on the left side, but was mute 
Fu et al.: Basilar Artery Territory Stroke Secondary to Invasive Fungal Sphenoid Sinusitis: A Case Report and Review of the Literature

and inattentive. Cranial nerve examination demonstrated a right gaze preference, and visual fields did not react to threat. Extraocular eye movements were intact. On the right side, there was decreased tone in the right arm, more so than in the right leg, and he did not move the upper or lower extremities, but withdrew in response to pain. The patient showed left-side antigravity without drift in either his arm or leg. Deep tendon reflexes were hyperactive without clonus throughout, with the right toe upgoing.

Two days after admission, antibiotics were discontinued after the Gram stain of the CSF was found to be negative and the cultures were negative after $24 \mathrm{~h}$. Acyclovir was also discontinued following a negative herpes simplex virus polymerase chain reaction. Coccidiomycoses antibodies for CSF and serum were both negative, WNV ELISA IgM and IgG tests were negative, and cultures and stains for acid-fast bacilli were negative, decreasing the likelihood of mycobacterial meningoencephalitis, WNV infection, or a coccidiomycosis infection. Parasitic infections such as strongyloidiasis or neurocysticercosis may give a similar clinical picture with eosinophilic CSF pleocytosis. However, he had always lived in Los Angeles and Arizona without traveling further afield and without a history of consuming raw snails or exotic foods. Therefore, parasitic infection was unlikely given his social history, negative travel history, and lack of risk factors.

MRI diffusion-weighted imaging demonstrated stroke in the basilar artery perforator and left posterior inferior cerebellar artery territory, including the left hemi-midbrain, upper pons, and left cerebellum (fig. 1a). Diffuse marrow signal abnormality within the clivus and surrounding central skull base with contiguous sinus disease was seen in addition to abnormal, peripheral meningeal enhancement and thickening surrounding the clivus. These findings suggested invasive fungal sinusitis. A head CT the following day also revealed evolution of the large infarct of the left cerebellum, pons, and midbrain, now involving the left red nucleus with destruction of the clivus (fig. 1b). High-density material was noted within the high sinuses. The diffuse marrow signal abnormality within the clivus and surrounding central skull base, with contiguous sinus disease, was also seen on the axial (fig. 1c) and coronal (fig. 1d) orientations of CT angiography (CTA), demonstrating sinusitis, likely of fungal etiology, invading through and destroying the clivus. The left superior cerebellar artery was not visualized on CTA.

Upon transfer to the neurology service 3 days after admission, the patient was awake, alert and oriented, able to say his name and respond to simple commands. Confrontation showed no visual field loss. His pupils were of equal size, round and reactive to light. He continued to have a right gaze preference. There was normal muscle bulk and tone with no tremor. 5/5 strength was found for his left upper and lower extremities and $0 / 5$ strength for his right upper and lower extremities. He responded to noxious stimuli throughout. Deep tendon reflexes were 3+ throughout and brisker on the left with no clonus. Due to the concern for fungal infection, ambisome $500 \mathrm{mg}$ i.v. was started with discontinuation of fluconazole therapy. Otolaryngology was consulted to evaluate the need for surgical drainage.

An endoscopic sinus surgery and left maxillary antrostomy, ethnoidectomy, sphenoidectomy, and biopsy revealed extensive polypoid change of the left maxillary, ethmoid, and sphenoid sinuses. Multiple biopsies were sent for pathologic evaluation, which revealed focally necrotic tissue with tissue-invasive fungal hyphae forms morphologically consistent with mucormycosis of the Rhizopus species on culture. The left clivus also showed focally necrotic fibrous tissue with fungal hyphae forms. A maxillofacial CT revealed findings most consistent with extensive fungal rhinosinusitis with skull base involvement. Liposomal amphotericin was continued as the principal antifungal drug with the addition of posaconazole (400 mg p.o. twice daily). Progressive neurological decline was noted as the mucormycosis progressed, and the patient no longer attempted to speak and did not follow commands. 
Fu et al.: Basilar Artery Territory Stroke Secondary to Invasive Fungal Sphenoid Sinusitis: A Case Report and Review of the Literature

A family meeting was held and a decision was made to focus care on comfort measures in a hospice facility. At discharge, all medications were discontinued and the patient remained comfortable and was not in any pain. A meeting with home hospice care was scheduled for that afternoon, and the patient was accepted to a hospice care facility. He passed away following the transfer to the hospice.

\section{Discussion}

We herein present the case of an invasive mucormycosis leading to stroke and death in a 38-year-old diabetic man. Some reports have described patients who developed this invasive fungal infection in the absence of diabetic ketoacidosis [3, 4], similarly to our patient. Previous research has more specifically reported fatal stroke occurring in those with diabetes mellitus without ketoacidosis who developed rhino-orbital-cerebral mucormycosis, also similar to our patient's clinical presentation [4]. Diabetes mellitus has been associated with a susceptibility to infection perhaps linked to its association with altered immune function, such as diminished T-cell responses, problems with humoral immunity, decreased neutrophil phagocytic ability and adherence to endothelial walls, and impaired function of monocytes and macrophages $[5,6]$. Acidosis and subsequent reduced serum inhibitory activity against Rhizopus in lower $\mathrm{pH}$ values in addition to hyperglycemia and greater availability of glucose to the pathogen contribute to a favorable environment for fungal growth, enabling angioinvasion with thrombosis and tissue necrosis [6]. Therefore, while the patient reported that he may not have had diabetic ketoacidosis, his diabetes mellitus may have provided a sufficiently favorable environment for the development of mucormycosis.

Other predisposing risk factors for this patient's presentation include his chronic sinusitis; this has been demonstrated in previous studies to increase the risk of stroke among patients diagnosed with chronic rhinosinusitis [7]. Such studies have suggested that chronic sinusitis can directly induce vasculopathy, resulting in stroke [8]. This condition may then be further exacerbated by superimposed infections involving intracerebral vessels, further increasing the risk of damage to these vessels and the occurrence of stroke. Other studies have suggested that the anatomic proximity of the brain to the sinuses may increase the risk of a perivascular inflammatory reaction, since an accumulation of proinflammatory cytokines is often found in the sinuses of patients with chronic sinusitis. These inflammatory mediators can result in local arterial thrombosis and an occlusion of the basilar and carotid arteries [8]. Exposure of intracerebral vessels to increased amounts of inflammatory mediators can also result in spasm of the vessels, which may lead to acute ischemic stroke [9]. Therefore, despite the lack of diabetic ketoacidosis and other factors related to immunodeficiency, this patient's diabetes mellitus may have provided a sufficiently favorable environment for the survival and growth of the fungal pathogen while his chronic sinusitis may have weakened the integrity of certain intracerebral vessels, predisposing them to invasive fungal infection and a subsequent thrombosis of the basilar artery.

Mucormycosis has been implicated in various types of cerebral involvement, such as hemorrhage, infarction, and ophthalmoplegia. The pathologic basis of the occurrence of cerebral ischemia and infarction in the setting of mucormycosis involves a combination of growth of the hyphae into the arterial lumen and direct endothelial injury resulting in mycotic emboli and more distal infarction of the vessels [4]. This mucormycosis-associated vasculopathy in combination with preexisting diabetic vasculopathy increases the risk of intracerebral infarction and acute ischemic stroke through thrombotic occlusion of major vessels of the brain, such as the carotid and basilar arteries. While previous reports of either 
Fu et al.: Basilar Artery Territory Stroke Secondary to Invasive Fungal Sphenoid Sinusitis: A Case Report and Review of the Literature

mucormycosis or sinusitis have often led to cavernous sinus thrombosis involving the carotid artery [3], our patient had a mucormycosis with involvement of the skull base that led to a left cerebellar and upper pontine stroke as a result of involvement of the basilar artery. This presentation more closely resembles previous cases of mucormycosis with skull base involvement and pontine infarction [10] or cases affecting the basilar artery [4].

Eosinophilic meningitis has been previously described in various fungal infections, such as secondary to allergic Aspergillus sinusitis, while eosinophilic CSF pleocytosis has been reported with cryptococcal and coccidioidal meningitis as well as acute cerebral phaeohyphomycosis [11]. Other mycoses, including candidiasis, histoplasmosis, blastomycosis, and mucormycosis, may affect the central nervous system but often do not produce eosinophilic inflammatory reactions in the meninges, except on rare occasions [11]. Our patient was unusual in that he presented with eosinophilic CSF pleocytosis with mucormycosis.

A prompt diagnosis of mucormycosis is crucial, with neuroimaging assuming a substantial role in the diagnostic process. While CT is the initial imaging method of choice for the detection of fungal intracerebral infection and invasion of vasculature, it may be nonspecific in the early stages of the disease [12]. CT is more effective in detecting bony erosion or destruction while MRI is more sensitive for detecting invasive soft tissue, extra-sinus spread, and vessel involvement [13]. While a CT scan was done in this patient to better understand the involved regions of the cerebellar stroke, a subsequent MRI revealed abnormal, peripheral meningeal enhancement and thickening surrounding the clivus, suggesting fungal invasion. The use of CT scan to detect bone destruction was useful as a later CTA revealed invasion and destruction of the clivus, confirming the likelihood of a fungal etiology. A definitive diagnosis is primarily achieved with endoscopic evaluation and biopsy with subsequent histological study that identifies characteristic features of the pathogen, such as extensive tissue necrosis or numerous and irregular broad, nonseptate hyphae that branch at right angles [14], as done in the case of this patient. The standard stains for histological study used are often hematoxylin and eosin, periodic acid-Schiff, and Gomori's methenamine silver. However, a drawback of this approach is a potential delay in treatment and increased morbidity and mortality of the disease given its lengthy and time-consuming nature [15].

Administration of amphotericin B has been the recommended treatment option and antifungal treatment of choice for mucormycosis [15], with posaconazole representing an acceptable therapeutic option as well [6]. Aggressive surgical debridement in the case of a rapid-growing, aggressive, and invasive infection is often also an essential component of treatment [2], but was not an option with this patient given the severity of the fungal invasion. In the case of this patient, fluconazole was initially administered to cover general fungal infection, but was subsequently switched to amphotericin B once the diagnosis of mucormycosis had been made. Posaconazole was added as an adjuvant therapy, similar to other reported cases of mucormycosis.

Survival rates for mucormycosis have been reported to be approximately $70 \%$ when amphotericin B and a rapid, aggressive surgical approach are combined [2]. Most cases of mucormycosis have a poor prognosis given the delay in diagnosis and treatment. Brain involvement, hemiparesis, bilateral sinus involvement, and facial necrosis are all associated with poor survival.

Since this patient presented to our clinic at the time of his basilar and left cerebellar artery stroke, the mucormycosis invasion into the cerebral vasculature was already severe. Earlier diagnosis at the time of his admission therefore may not have had a significant impact on the course and prognosis of his disease, though in general, mucormycosis is a medical emergency and merits rapid diagnosis and treatment whenever possible. While many of the diagnostic and treatment approaches in the management of this case corroborate estab- 
Fu et al.: Basilar Artery Territory Stroke Secondary to Invasive Fungal Sphenoid Sinusitis: A Case Report and Review of the Literature

lished approaches towards managing a case of rhinocerebral mucormycosis, the severity of the disease stage was so that this care was not enough to significantly improve his prognosis or the final outcome. This case was also unique in that the patient was a middle-aged, immunocompetent man with no significant predisposing risk factors, aside from his diabetes and sinusitis, who presented with primarily nonspecific symptoms leading to stroke, such as fatigue, weakness, and nausea.

Clinicians should be aware of invasive sinusitis as a rare cause of stroke in diabetics. Once there has been an invasion into the subarachnoid space and basal arteries of the brain, the prognosis is very poor. The key to improvement of the outcomes is early recognition and treatment, and examination of the sinuses on neuroimaging in all cases of stroke is vital.

\section{References}

1 Gen R, et al: Rhino-orbito-cerebral mucormycosis in patients with diabetic ketoacidosis. J Craniofac Surg 2013;24:e144-e147.

2 Bhadada S, et al: Rhino-orbital-cerebral mucormycosis in type 1 diabetes mellitus. Indian J Pediatr 2005;72:671-674.

-3 Turunc T, et al: Eleven cases of mucormycosis with atypical clinical manifestations in diabetic patients. Diabetes Res Clin Pract 2008;82:203-208.

4 Thajeb P, Thajeb T, Dai D: Fatal strokes in patients with rhino-orbito-cerebral mucormycosis and associated vasculopathy. Scand J Infect Dis 2004;36:643-648.

5 Peleg AY, et al: Common infections in diabetes: pathogenesis, management and relationship to glycaemic control. Diabetes Metab Res Rev 2007;23:3-13.

-6 Spellberg B, Edwards J Jr, Ibrahim A: Novel perspectives on mucormycosis: pathophysiology, presentation, and management. Clin Microbiol Rev 2005;18:556-569.

$\checkmark 7$ Kang JH, et al: Chronic rhinosinusitis increased the risk of stroke: a 5-year follow-up study. Laryngoscope 2013;123:835-840.

8 Perez Barreto M, et al: Sinusitis and carotid artery stroke. Ann Otol Rhinol Laryngol 2000;109:227-230.

-9 Tokushige E, et al: Localization of IL-1 beta mRNA and cell adhesion molecules in the maxillary sinus mucosa of patients with chronic sinusitis. Laryngoscope 1994;104:1245-1250.

$\checkmark 10$ Calli C, et al: Isolated pontine infarction due to rhinocerebral mucormycosis. Neuroradiology 1999;41:179181.

11 Graeff-Teixeira C, da Silva AC, Yoshimura K: Update on eosinophilic meningoencephalitis and its clinical relevance. Clin Microbiol Rev 2009;22:322-348.

$\checkmark 12$ Dhiwakar M, Thakar A, Bahadur S: Improving outcomes in rhinocerebral mucormycosis - early diagnostic pointers and prognostic factors. J Laryngol Otol 2003;117:861-865.

13 Herrera DA, et al: Imaging findings of rhinocerebral mucormycosis. Skull Base 2009;19:117-125.

14 Singh P, et al: Disseminated mucormycosis and orbital ischaemia in combination immunosuppression with a tumour necrosis factor alpha inhibitor. Clin Experiment Ophthalmol 2007;35:275-280.

15 O'Neill BM, et al: Disseminated rhinocerebral mucormycosis: a case report and review of the literature. J Oral Maxillofac Surg 2006;64:326-333. 
Fu et al.: Basilar Artery Territory Stroke Secondary to Invasive Fungal Sphenoid Sinusitis: A Case Report and Review of the Literature

Table 1. Lumbar puncture results and CSF values

\begin{tabular}{|c|c|c|c|c|}
\hline & $\begin{array}{l}\text { Day } 1 \\
(15: 31)^{a}\end{array}$ & $\begin{array}{l}\text { Day } 1 \\
(17: 21)^{a}\end{array}$ & $\begin{array}{l}\text { Day 14 } \\
(19: 18)^{a}\end{array}$ & $\begin{array}{l}\text { Day } 14 \\
(19: 18)^{a}\end{array}$ \\
\hline Color & colorless & colorless & colorless & colorless \\
\hline Clarity & clear & clear & clear & clear \\
\hline $\mathrm{RBC}$ count, cells $/ \mathrm{mm}^{3}$ & 16 & 3 & 7 & 1 \\
\hline WBC count, cells $/ \mathrm{mm}^{3}$ & HP 400 & HP 365 & HP 194 & HP 187 \\
\hline Lymphocytes, \% & 27 & 24 & 35 & 36 \\
\hline Mono/histiocytes, \% & 5 & 5 & 8 & 10 \\
\hline Eosinophils, \% & 68 & 71 & 57 & 54 \\
\hline Glucose, mg/dl & 117 & $\mathrm{~N} / \mathrm{A}$ & $\mathrm{N} / \mathrm{A}$ & 83 \\
\hline Protein, mg/dl & 63 & $\mathrm{~N} / \mathrm{A}$ & $\mathrm{N} / \mathrm{A}$ & 59 \\
\hline
\end{tabular}

HP = Hematopoietic.

a Time of lumbar puncture. 
Fu et al.: Basilar Artery Territory Stroke Secondary to Invasive Fungal Sphenoid Sinusitis: A Case Report and Review of the Literature
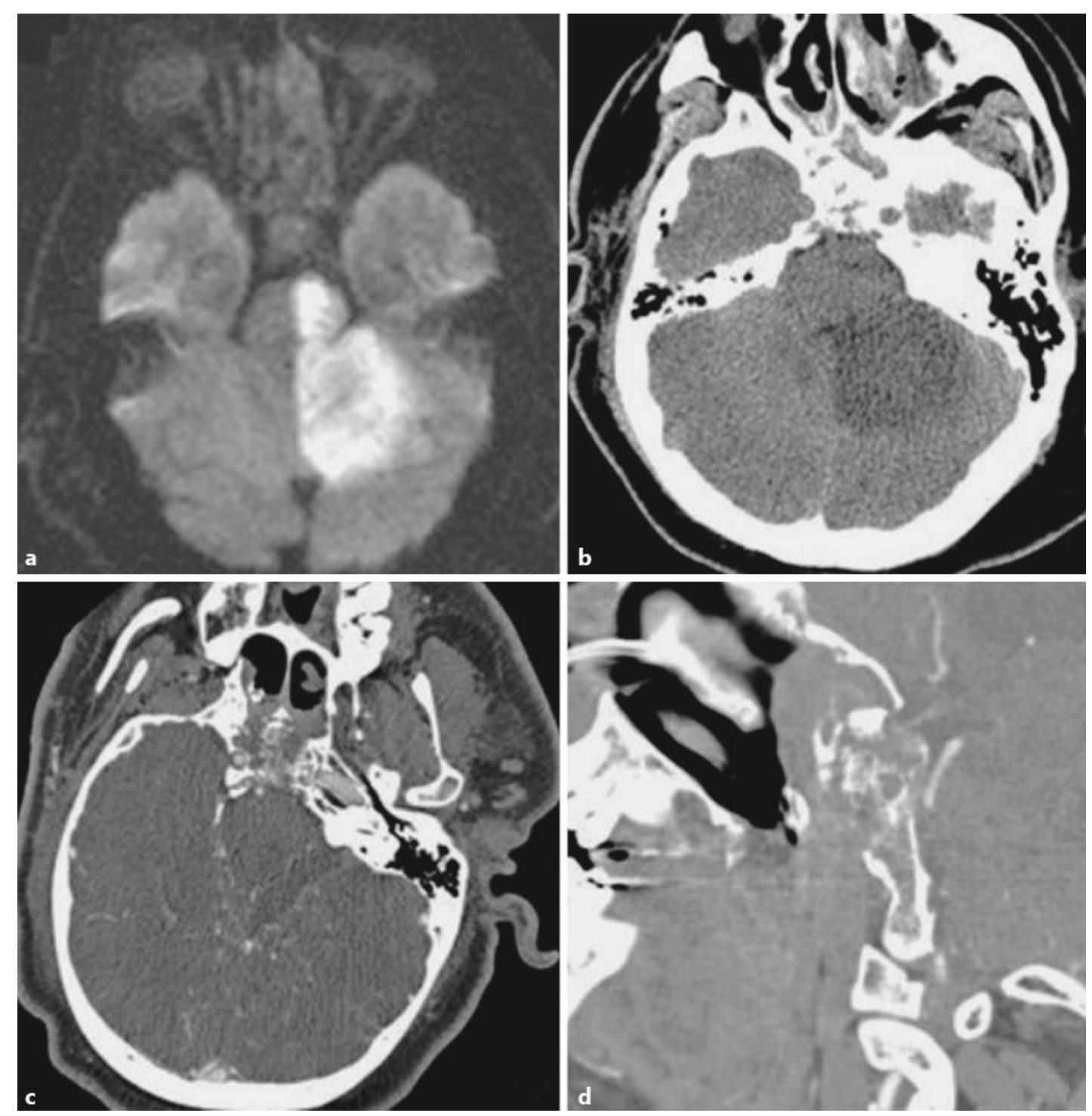

Fig. 1. a MRI diffusion-weighted imaging demonstrates stroke in the basilar artery perforator and left posterior inferior cerebellar artery territory, including the left hemi-midbrain, upper pons, and left cerebellum. b A head CT reveales evolution of a large infarct of the left cerebellum, pons, and midbrain, now involving the left red nucleus with destruction of the clivus. Diffuse marrow signal abnormality within the clivus and surrounding central skull base, with contiguous sinus disease, is seen on the axial (c) and coronal (d) orientations of CT angiography, demonstrating sinusitis, likely of fungal etiology, invading through and destroying the clivus. 